
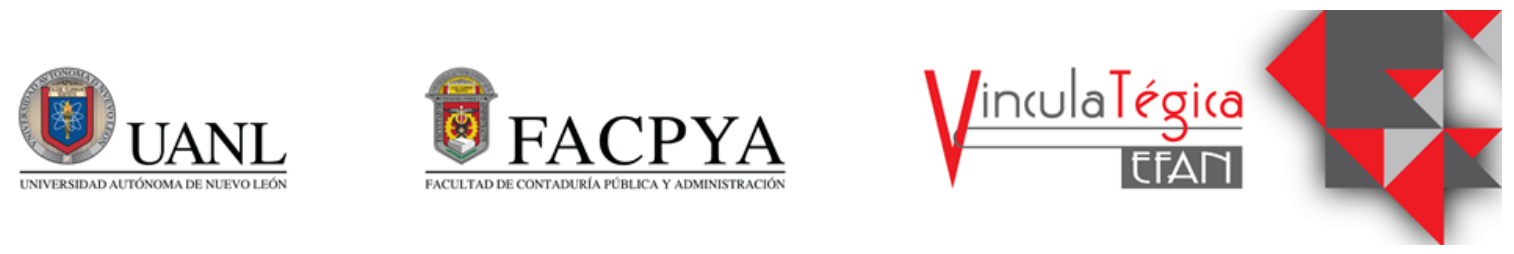

\title{
El liderazgo en organizaciones públicas de Ciudad Victoria (Tamaulipas)
}

\author{
Lázaro Castillo Hernández ${ }^{1}$, Jesús Lavín Verástegui ${ }^{2}$ y Maritza Álvarez Herrera ${ }^{3}$ \\ ${ }^{1}$ Universidad Autónoma de Tamaulipas, lcastilloh@docentes.uat.edu.mx, Centro Universitario Adolfo López \\ Mateos, C.P.87149, Ciudad Victoria, Tamaulipas, 8343181800 ext. 2493 \\ ${ }^{2}$ Universidad Autónoma de Tamaulipas, jelavin@docentes.uat.edu.mx, Centro Universitario Adolfo López \\ Mateos, C.P.87149, Ciudad Victoria, Tamaulipas, 8343181800 ext. 2493 \\ ${ }^{3}$ Universidad Autónoma de Tamaulipas, ahmaritza@gmail.com, Centro Universitario Adolfo López Mateos, \\ C.P.87149, Ciudad Victoria, Tamaulipas, 8343181800 ext. 2493
}

Información del artículo revisado por pares

Fecha de aceptación: junio-2021

Fecha de publicación en línea: diciembre-2021

DOI: https://doi.org/10.29105/vtga7.2-20

\section{Resumen}

Las tendencias de la nueva administración pública es propiciar un mejor servicio a la ciudadanía basado en resultados, para ello toma de gran relevancia el papel que juegan los líderes de las organizaciones en cuanto a sus capacidades y diferentes estilos de realizar su trabajo que motive a los seguidores al cumplimiento de sus compromisos y mejor desempeño para lograr los objetivos trazados. En este sentido, la presente investigación tiene como objetivo identificar los estilos de liderazgo y sus componentes en organizaciones públicas de Ciudad Victoria, Tamaulipas. La investigación se realizó bajo un enfoque cuantitativo con diseño no experimental y de corte transversal. Se aplicaron 106 cuestionarios a personal administrativo y de confianza del sector público. Utilizando la técnica de Análisis Factorial Exploratorio (AFE) se identificaron los componentes de los tres estilos de liderazgos transformacional (3), transaccional (2) y unidimensional para laissez faire. Los resultados obtenidos contribuyen desde la academia a la generación de

\begin{abstract}
The trends of the new public administration is to promote a better service to citizens based on results, for this the role played by the leaders of the organizations in terms of their capacities and different styles of carrying out their work that motivates the followers to fulfill their commitments and better performance to achieve the objectives set. In this sense, the present research aims to identify leadership styles and their components in public organizations in Ciudad Victoria, Tamaulipas. The research was carried out under a quantitative approach with a non-experimental and cross-sectional design. 106 questionnaires were applied to administrative and trust personnel of the public sector. Using the Exploratory Factor Analysis (EFA) technique, the components of the three leadership styles transformational (3), transactional (2) and onedimensional for laissez faire were identified. The results obtained contribute from the academy to the generation of knowledge and proposals for improvement in the performance of the leaders of the organizations for the good of society.
\end{abstract}


conocimiento y propuestas de mejora en el desempeño de los dirigentes de las organizaciones por el bien de la sociedad.

Palabras clave: Liderazgo, Organización, Líder.

Códigos JEL:M12

\section{INTRODUCCIÓN}

En el actual contexto mundial, los procesos de globalización han impulsado la competitividad de las organizaciones, de tal manera que los gerentes se ven obligados afrontar cambios donde ponen en práctica sus habilidades de liderazgo a fin de responder eficaz y eficientemente a las exigencias del entorno e impulsar el buen funcionamiento del ente que dirigen. Ante ello, el líder se presume como un agente crucial de cambio y desarrollo organizacional (Dinwoodie, Pasmore, Quinn y Rabin, 2015; Jun y Yazdanifard, 2015; Vos y Rupert, 2018), además de ser considerado un actor fundamental en el logro de la excelencia y gestión de la calidad (González, Carrión y Palacios, 2015; Tracy (2015).

Es a través de las habilidades del líder que la organización busca alcanzar las metas planeadas, ello debido a la capacidad que tiene el líder para influir en sus seguidores, fomentando el desarrollo de sus habilidades personales como la creatividad, participación y crecimiento (Perdomo y Prieto, 2009). Es decir, es el líder un generador de valor para que la organización obtenga los resultados esperados y cumpla sus objetivos, debido a que el liderazgo es un proceso de influencia capaz de generar una movilización de los intereses entre los individuos, al mismo tiempo que ayuda a enriquecer las habilidades de los involucrados en un esquema de desarrollo empresarial en el cual también crecen sus integrantes.

Por su parte Fernández (2014) comenta que los líderes son personas únicas, día tras día se enfrentan a nuevos retos y exigencias que inconscientemente los obligan a elegir estilos de liderazgo que les ayuden a dar respuesta a las necesidades organizacionales como la innovación, la diversidad cultural, la gestión del conocimiento, la armonía a entornos flexibles, fortuitos y la crisis de valores que
Key words: Leadership, Organization, Leader.

JEL Codes: M12 conduce a la crisis económica, entre otras variables de impacto organizacional.

Sin embargo, es importante señalar que, ante los diversos estilos que puede adoptar un líder, los seguidores forman percepciones que impactan en sus comportamientos y actitudes, además de regular la autoridad que el líder tiene sobre ellos, por lo que resulta trascendental para los dirigentes organizacionales, elegir adecuadamente el estilo de liderazgo que desean adoptar, ya que la ausencia de un liderazgo eficaz pudiera ocasionar, entre otras cosas, una deficiente comunicación entre los trabajadores, ambientes laborales turbios, una alta rotación de personal, bajo rendimiento laboral y la desmotivación por parte de los integrantes de la organización para agregar valor a los productos o servicios que ofrecen en cada una de las organizaciones en las que colaboran (Hernández, Hernández y Mendieta, 2013; Rivera y Conrado, 2016).

Es bajo este sentido que la presente investigación tiene como objetivo identificar los estilos de liderazgo y sus componentes en organizaciones públicas de Ciudad Victoria, Tamaulipas, considerando para tal efecto los estilos transaccionales, transformacional y laissez faire del modelo multifactorial de Bass y Avolio (1990), enfocado al seguidor. Así mismo esta investigación busca obtener información que sea de utilidad para los tomadores de decisiones respecto a la mejora en los estilos de liderazgo implementados en sus organizaciones.

\section{MARCO TEÓRICO}

\subsection{Conceptos y antecedentes}

El liderazgo, es una de las variables con mayor presencia en los estudios organizacionales debido al impacto que genera en las conductas de la ciudadanía organizacional.

Según Noriega (2008) la primera 
investigación empírica del liderazgo fue presentada en 1904 durante la Primera Guerra Mundial, y a partir de este punto se han presentado innumerables estudios que tratan este importante concepto.

Por definición, el liderazgo se puede conceptualizar como la capacidad interpersonal de un individuo para influir en los demás (Aguirre, Serrano y Sotomayor, 2017; Enderica, D’Armas, Bermeo, López, Tinoco y Carrión, 2018; Escandón y Hurtado, 2016; Gómez, 2016).

Así mismo es esencialmente un proceso natural de persuasión entre el líder y sus seguidores, consecuencia de un transcurso de percepción social, donde las conductas y características del líder le brindan los atributos y cualidades necesarias para ser visto como tal frente a los otros miembros de la organización (Lupano y Castro, 2006).

En este contexto, García (2011) comenta que algunos líderes pueden influir sobre otros según sus cargos o puestos, otros en cambio pueden influir de acuerdo con sus características y actitudes, las cuales generan identificación y entusiasmo en los seguidores, siendo esta última condición la especialmente aceptada en el papel de un buen líder, puesto que de él se espera sea facilitador de prácticas de cooperación organizacional, con capacidad de empuje y una actitud proactiva para optimizar el ímpetu de sus seguidores en beneficio de la comunidad organizacional (Barahona, Cabrera y Torres, 2011; Gómez, 2006).

Así mismo, es importante tener presente que el liderazgo es un fenómeno que no solo depende directamente de las personas que lo ejercen, también se ve afectado por el medio en que se desenvuelve y de la organización en el que se presenta (Barahona, et al, 2011).

En este contexto se puede comentar adicionalmente que, a lo largo de su estudio, el tema del liderazgo ha sido abordado desde diferentes perspectivas y teorías, donde cada una de ellas postula la idea de cómo el líder se debe comportar y las características que debe poseer para ser exitoso y eficiente (García, 2015).

\subsection{Enfoques de liderazgo}

En función de lo anteriormente descrito, se presenta los principales enfoques que se han planteado para analizar las conductas del líder al interior de las organizaciones, enfoques que han sido clasificados según los elementos que contempla el proceso de liderazgo y las características que posee el líder.

Como lo comentan los diversos autores que tratan el tema de liderazgo, existen variedad de conceptualizaciones teóricas que explican los comportamientos del líder y su impacto en la organización. Sin embargo, es de resaltar la teoría del liderazgo colectivo, a través del establecimiento de una visión y una misión compartida (Almirón, Tikhomirova, Trejo y García, 2015; Cruz, Salanova y Martínez, 2013; Pérez, Jiménez y Robo, 2017).

Por su parte Stogdill (1948), refiere que los lideres poseen cualidades y características personales que distinguen a los lideres de quien no lo son y comenta que los lideres nacen, no se hacen. Pero también se pueden tener dos enfoques de líder, uno de ellos el que se centra en la producción y el otro el que se centra en las personas.

En el mismo contexto también se pueden comentar los estudios de Fiedler (1964), quien asevera que, si se desea tener un liderazgo eficaz, este debe cambiar de acuerdo con las necesidades de la situación tratando de adaptarse al medio en el que se requiere un líder.

Este tema del liderazgo situacional también expresa que el líder es afectado por la situación en la que se encuentra y tiene que adaptarse debido a que el líder es el responsable de guiar a sus seguidores, y con ello ayudar a cumplir los objetivos prometidos al personal integrante de las organizaciones en las que se desempeñan (Hersey y Blanchard, 1974).

Por su parte Bass y Avolio (1990) refieren un conjunto de dimensiones con la finalidad de estudiar el liderazgo y obtener indicadores que midan este fenómeno; con base en lo anterior, desarrollaron el modelo de liderazgo de rango completo (MLRC), el cual incluye tres dimensiones, que son: liderazgo transaccional, liderazgo transformacional y laissez-faire. 


\subsubsection{Liderazgo Transaccional}

En este tipo de liderazgo se observa un proceso transaccional, en el cual la relación de liderazgo supone un intercambio que puede ser económico o psicológico entre la figura del líder y el empleado, de modo que ambos elementos sean beneficiados de dicha transacción.

Esta definición Bass (1985) lo identifica como un estilo de liderazgo que se concentra en transacciones interpersonales entre los gerentes (líder) y los empleados, donde cada parte es responsable de una acción por la cual obtienen una contraprestación.

Por otro lado, Sosik y Dionne (1997) comentan que en el liderazgo transaccional los líderes se acercan a los seguidores con la intención en intercambiar una cosa por otra, lo que nos afirma que es un proceso de dar y recibir entre las partes interesadas.

El liderazgo transaccional tiene la característica principal de que guía por medio de intercambios sociales, este tipo de liderazgo funciona por medio de recompensas a cambio del cumplimiento de metas y de productividad (Robbins 2010). Así mismo Hemphil y Coons (1954) comenta que el liderazgo transaccional representa aquellos intercambios en la influencia del líder y el subordinado lo cual deriva en algo de valor para la organización.

Por su parte, Rejas y Ponce (2008) definen al liderazgo transaccional como aquel en el cual los seguidores se motivan en base a los beneficios esperados por el logro de las metas o tareas encomendadas, definición que es apoyada por Alonso, Saboya y Guirado (2010) quienes mencionan que el liderazgo transaccional se basa en el intercambio de recompensas entre el líder los miembros de su equipo.

Pero también Bass (1997) afirma que el liderazgo transaccional es el proceso de construir en base a intercambios, el líder ofrece recompensas, o en su caso castigos, a cambio del desempeño deseado, el comportamiento y finalizar las tareas.

Este tipo de liderazgo es posiblemente el más estudiado hasta la fecha, y está estrechamente relacionado con los resultados esperados por los individuos (Liu J. y Siu, O., 2010). También se puede mencionar que existen tres componentes del liderazgo transaccional: recompensa contingente, gestión activa por excepción y gestión pasiva por excepción (Bass, 1997).

Donde la recompensa contingente habla sobre el desempeño de los subordinados en el cual está asociado con recompensas contingentes o relaciones de intercambio (Bass, 1997). De acuerdo con Bass y Riggio (2006) la recompensa contingente crea acuerdos con los empleados donde acceden a realizar cierto trabajo en un límite de tiempo a cambio de recompensas ya esperadas por los seguidores.

En la gestión activa por excepción los lideres monitorean el desempeño de los seguidores y toman acciones correctivas con el fin de que se cumplan los objetivos (Bass, 1997). Así mismo, supervisa intensamente a los empleados, identifica sus errores y fallos, $\mathrm{y}$ es entonces cuando toma acciones correctivas (Bass y Riggio, 2006), y por último la gestión pasiva en la cual el líder no interviene hasta que el problema se convierta en algo serio (Bass, 1997), y solamente interfiere en el trabajo de los empleados cuando los errores o fallos ocurren (Bass y Riggio, 2006).

\subsubsection{Liderazgo Transformacional}

Los autores Bass y Avolio (1990) establecen que este modelo de liderazgo se caracteriza por el intercambio entre líder y seguidor, lo cual provoca que los seguidores sufran un cambio en sus necesidades, creencias y valores. Así mismo siguieren que el estilo de liderazgo transformacional sería una extensión del liderazgo transaccional.

Esto en base a lo que Bass y Avolio (1990) plantean, que los lideres transformacionales pueden actuar, en ciertos casos, con un estilo de liderazgo transformacional, pero los líderes transaccionales no pueden hacerlo con un estilo transformacional.

El liderazgo transformacional se distingue porque estimula e inspira a los seguidores a alcanzar resultados 
extraordinarios, es importante mencionar que este liderazgo produce niveles de esfuerzo mayores a los de otro estilo. (Robbins y Coulter, 2010)

Por su parte Sosik y Dionne (1997) también comentan que el estilo de liderazgo transformacional es el más efectivo, esto debido a que los lideres transformacionales crean una relación mutua que convierte al seguidor en líder y puede convertir al líder en un agente moral. En este estilo de liderazgo, el líder se caracteriza por ser proactivo, despertar conciencia en sus seguidores y trascender sobre el interés colectivo, esto con el fin de llevar a sus seguidores a obtener resultados extraordinarios. (Antonakis, J., Avolio, B. y Sivasubramaniam, N., 2003)

Bass (1985) establece 4 factores o características del liderazgo transformacional las cuales son en primer término la Influencia idealizada, esta se subdivide en dos tipos, atributos y por conducta, es decir, los seguidores idealizan y simulan el comportamiento del líder (Bass,1985), la siguiente es la motivación inspiracional que se refiere a que los seguidores están motivados por la consecución de un objetivo en particular (Bass,1985), posteriormente la estimulación intelectual en la cual los seguidores son animados a romper con los paradigmas y se les propone cuestionar sus valores, creencias y expectativas actuales (Bass, 1985) y finalmente la consideración individualizada donde las necesidades de los seguidores son dirigidas tanto a un nivel individual $\mathrm{y}$ equitativamente (Bass y Avolio, 1997).

\subsubsection{Liderazgo Laissez-Faire}

Por último, Sosik y Dionne (1997) comentan que el estilo de liderazgo LaissezFaire es el más inefectivo y pasivo, también lo llaman como el no liderazgo, y se caracteriza por retrasos en la acción, ausencia e indiferencia del líder.

Bass (1985) comenta que los líderes

que siguen la teoría de laissez-faire generalmente evitan tomar decisiones críticas o cambios, por otro lado, Eglay, JohannsenSchmidt y Van Engen (2003) hablan de que los líderes que utilizan el estilo de liderazgo laissez-faire evitan tomar responsabilidades en circunstancias críticas. Un sinónimo de este estilo de liderazgo es el liberal, propuesto por Koontz y Weihrich (1998) donde afirman que el líder hace uso muy reducido de su poder, ya que les concede a sus seguidores un alto grado de independencia en sus actividades.

Ma y Tsui (2015) explican que una de las justificaciones del uso del estilo laissezfaire es que cuando los líderes no hacen nada, la mayoría de la gente es libre de seguir su honestidad y su naturaleza, pero cuando los líderes establecen reglas precisas para regular y castigar, algunas personas pueden llegar a convertirse en "ladrones astutos", por lo tanto, no actuar es la mejor manera para permitir a la gente hacer las cosas correctamente.

\subsection{Modelo de Liderazgo de Bass y Avolio}

Bass (1985) refiere que las teorías de liderazgo se concentraban en las metas del empleado, y en la manera en que el líder recompensaba o sancionaba el comportamiento del seguidor, además sugirió que era necesario un cambio de paradigma para entender la influencia de los líderes.

El desarrollo de modelo de seis factores de Bass (Forma 1) se basó originalmente en los resultados preliminares de una encuesta realizada a oficiales de la armada de los Estados Unidos. En base al trabajo anterior, Bass y Avolio (1997) propusieron un modelo de liderazgo llamado "Full-range of Leadership Development" (FRLD). Este modelo brinda una descripción de los estilos de liderazgo específicos en términos de efectividad, grado de actividad y frecuencia de utilización.

La gran mayoría de las publicaciones e investigaciones empíricas sobre el tema del liderazgo han utilizado el Cuestionario Multifactorial del Liderazgo, que fue desarrollado por Bass y sus colegas (Bass, 1991).

Sin embargo, Yukl (1999) analizó cómo se medía el liderazgo transaccional, y afirmó que el liderazgo transaccional incluye una colección diversa de comportamientos del líder que carecen de algún denominador común. Otra de las críticas que se le han hecho 
a este cuestionario es que las primeras versiones no diferenciaban entre dos de las dimensiones del liderazgo transaccional, gestión por excepción pasiva y gestión por excepción activa (Hinkin y Schriesheim, 2008).

El modelo completo de Bass y Avolio (1990) incluye tres variables de resultados, Satisfacción, la cual está integrada por las acciones del líder, que provocan gratificación en el grupo , lo cual determina como se sienten lo seguidores, los estilos de liderazgo anteriormente comentados se muestran en la tabla 1.

Tabla 1. Enfoques de los estilos de liderazgo

\begin{tabular}{|c|c|c|}
\hline Estilos de liderazgo & Categoría & Descripción \\
\hline \multirow[t]{3}{*}{$\begin{array}{l}\text { Liderazgo } \\
\text { transaccional }\end{array}$} & $\begin{array}{l}\text { Recompensa } \\
\text { por } \\
\text { contingencia }\end{array}$ & $\begin{array}{l}\text { Crea acuerdos con los empleados donde acceden a } \\
\text { realizar cierto trabajo en un límite de tiempo a cambio } \\
\text { de recompensas. }\end{array}$ \\
\hline & $\begin{array}{l}\text { Gestión activa } \\
\text { por excepción }\end{array}$ & $\begin{array}{l}\text { Supervisa intensamente a los empleados, identifica sus } \\
\text { errores y fallos, y es entonces cuando toma acciones } \\
\text { correctivas }\end{array}$ \\
\hline & $\begin{array}{l}\text { Gestión pasiva } \\
\text { por excepción }\end{array}$ & $\begin{array}{l}\text { Solamente interfiere en el trabajo de los empleados } \\
\text { cuando los errores o fallos ocurren }\end{array}$ \\
\hline \multirow[t]{5}{*}{$\begin{array}{l}\text { Liderazgo } \\
\text { transformacional }\end{array}$} & $\begin{array}{l}\text { Influencia } \\
\text { idealizada }\end{array}$ & $\begin{array}{l}\text { Por atributos. Es la capacidad de un líder en influir en } \\
\text { los seguidores proporcionando en sentido de visión y } \\
\text { misión, inspira orgullo, respeto, búsqueda de logro y de } \\
\text { confianza, incrementa el optimismo. }\end{array}$ \\
\hline & & $\begin{array}{l}\text { Por conducta. Establece ejemplo de comportamiento } \\
\text { seguidor a través de acciones propias, subordinados } \\
\text { miran al comportamiento del líder de orientación de su } \\
\text { propio comportamiento }\end{array}$ \\
\hline & $\begin{array}{l}\text { Motivación } \\
\text { inspiracional }\end{array}$ & $\begin{array}{l}\text { Se refiere a que los seguidores están motivados por la } \\
\text { consecución de un objetivo en particular. }\end{array}$ \\
\hline & $\begin{array}{l}\text { Estimulación } \\
\text { intelectual }\end{array}$ & $\begin{array}{l}\text { Los seguidores son animados a romper con los } \\
\text { paradigmas y se les propone cuestionar sus valores, } \\
\text { creencias y expectativas actuales. }\end{array}$ \\
\hline & $\begin{array}{l}\text { Consideración } \\
\text { individualizada }\end{array}$ & $\begin{array}{l}\text { Las necesidades de los seguidores son dirigidas tanto a } \\
\text { un nivel individual y equitativamente }\end{array}$ \\
\hline Laissez-faire & Laissez-faire & $\begin{array}{l}\text { Pasa la toma de decisiones importantes } \\
\text { a responsabilidad de los subordinados, renuencia a } \\
\text { expresar puntos de vista sobre cuestiones importantes o } \\
\text { controvertidas. }\end{array}$ \\
\hline
\end{tabular}

Fuente: Elaboración a partir de autores citados.

\section{MÉTODO}

Este contiene 36 afirmaciones, las cuales tienen como fin identificar entre los estilos de liderazgo transformacional, transaccional o
La presente investigación se desarrolló bajo el enfoque cuantitativo, tipo transversal y diseño no experimental, de acuerdo con Cuadras (2007) el investigador no tiene control sobre las variables.

Para recabar la información se aplicó el Cuestionario Multifactorial del Liderazgo (MLQ, por sus siglas en inglés) que fue desarrollado por Bass y Avolio (1990). 
laissez faire, y cuenta con opciones de respuesta del estilo Likert de cinco puntos entre los rangos de $1=$ Nunca y $5=$ Siempre. Dada la dificultad de poder acceder a las diferentes dependencias debido a la contingencia por el Covid-19, cabe señalar que se determinó una muestra no probabilística y a conveniencia de 106 empleados del sector público. Los datos recabados fueron capturados para conformar una base de información en software estadístico SPSS versión 23. Posteriormente se analizan los datos generales con medidas de tendencia central de la estadística descriptiva, que de acuerdo con Bernal (2010) es la rama que recolecta, analiza y caracteriza un conjunto de datos (frecuencias y porcentajes).

Para identificar los factores que caracterizan a cada uno de los estilos de liderazgo, se aplicó la técnica de análisis factorial, que de acuerdo con Hair, Anderson, Tatharn, \& Black (1999) es un método estadístico multivariante cuyo propósito principal es definir la estructura subyacente en una matriz de datos, donde se puede identificar las dimensiones y el grado en que se justifica cada variable por cada dimensión. Dado el tamaño de la muestra se tomaron los ítems que cumplían con la carga factorial por encima de 0.55 según (Hair, et al. 1999).

\section{RESULTADOS}

Los resultados obtenidos en las variables sociodemográficas a partir de los datos proporcionado por los encuestados se encontraron que el $49.1 \%$ fueron mujeres y $50.9 \%$ hombres. Se encontró también que el $59.4 \%$ ocupan cargos de administrativo y jefe de área, un $29.3 \%$ es personal de confianza, así como un porcentaje bajo $(6.6 \%)$ de personal operativo y un $4.7 \%$ señaló desempeñar otros cargos.

En lo referente a la antigüedad el $52.8 \%$ tiene menos de 5 años, un $21.7 \%$ entre 6 y 10 años y el $25.5 \%$ de los encuestados laboran hace más de 10 años en la organización. Alrededor del $53.7 \%$ de los encuestados tiene más de 31 años de edad, un $42.5 \%$ tiene entre 21 a 30 años de edad y un porcentaje bajo $(3.8 \%)$ se ubican en el rango de 18 a 20 años.

El $54.7 \%$ de los empleados tiene escolaridad de nivel licenciatura y un $25.5 \%$ estudios de posgrado, sólo un $19.8 \%$ señaló contar con educación hasta nivel de preparatoria.

Se obtuvieron las estructuras subyacentes de los diferentes estilos de liderazgo al aplicar la técnica de análisis factorial exploratorio (AFE). En lo referente al estilo transaccional (tabla 2) resultaron dos factores con el $56.53 \%$ de la varianza total explicada (Gestión activa y Recompensa; Gestión Pasiva).

Se obtuvieron los valores adecuados para el uso de este método de reducción de datos, ya que se obtuvo un KMO mayor a 0.70 de acuerdo con Hair et al. (2008).

La significancia del test de Bartlet se encuentran en los valores aceptados ( $\mathrm{p}$-valor $=$ 0.000) y solamente fue eliminado un ítem debido a que no cumplía con la carga factorial de 0.55 . Como se puede observar en la tabla 2 en lo referente a la consistencia interna basado en el Alpha de Cronbach se obtuvo un valor por encima de 0.7 por lo que la confiabilidad de la escala se considera aceptable.

En a Tabla 3 se presentan los resultados obtenidos para el constructo liderazgo transformacional, cabe mencionar que se eliminaron 2 ítems (15 Dedica tiempo a enseñar y orientar y el 34 Enfatiza la importancia de tener una misión compartida), al no cumplir con la carga factorial adecuada (varianza total explicada 69.1\%). (Gestión activa y Recompensa; Gestión Pasiva).

Se obtuvieron los valores adecuados para el uso de este método de reducción de datos, ya que se obtuvo un KMO mayor a 0.90 y p-valor $=0.000$.

Como se puede observar en la tabla 3 en lo referente a la consistencia interna basado en el Alpha de Cronbach se obtuvo un valor por encima de 0.9 por lo que la confiabilidad de la escala se considera excelente según George y Mallery (2003, p. 231).

En lo referente a la variable Laissez-faire se puede observar (tabla 4) que en su medición se obtuvo un resultado unidimensional, ya que se obtuvo un solo factor mediante la aplicación 
de la técnica de análisis factorial exploratorio (AFE) que explica el $56.20 \%$ de la varianza., así como un KMO adecuado (superior a 0.70 ).

Tabla 2. Estructura del estilo de liderazgo: transaccional

\begin{tabular}{|c|c|c|c|c|c|}
\hline $\begin{array}{c}\text { Estilo de } \\
\text { Liderazgo }\end{array}$ & Factor & Descripción del reactivo & $\begin{array}{c}\text { Carga } \\
\text { factorial }\end{array}$ & $\begin{array}{c}\text { Análisis } \\
\text { factorial } \\
\text { exploratorio }\end{array}$ & $\begin{array}{l}\text { Alpha de } \\
\text { Cronbach }\end{array}$ \\
\hline
\end{tabular}

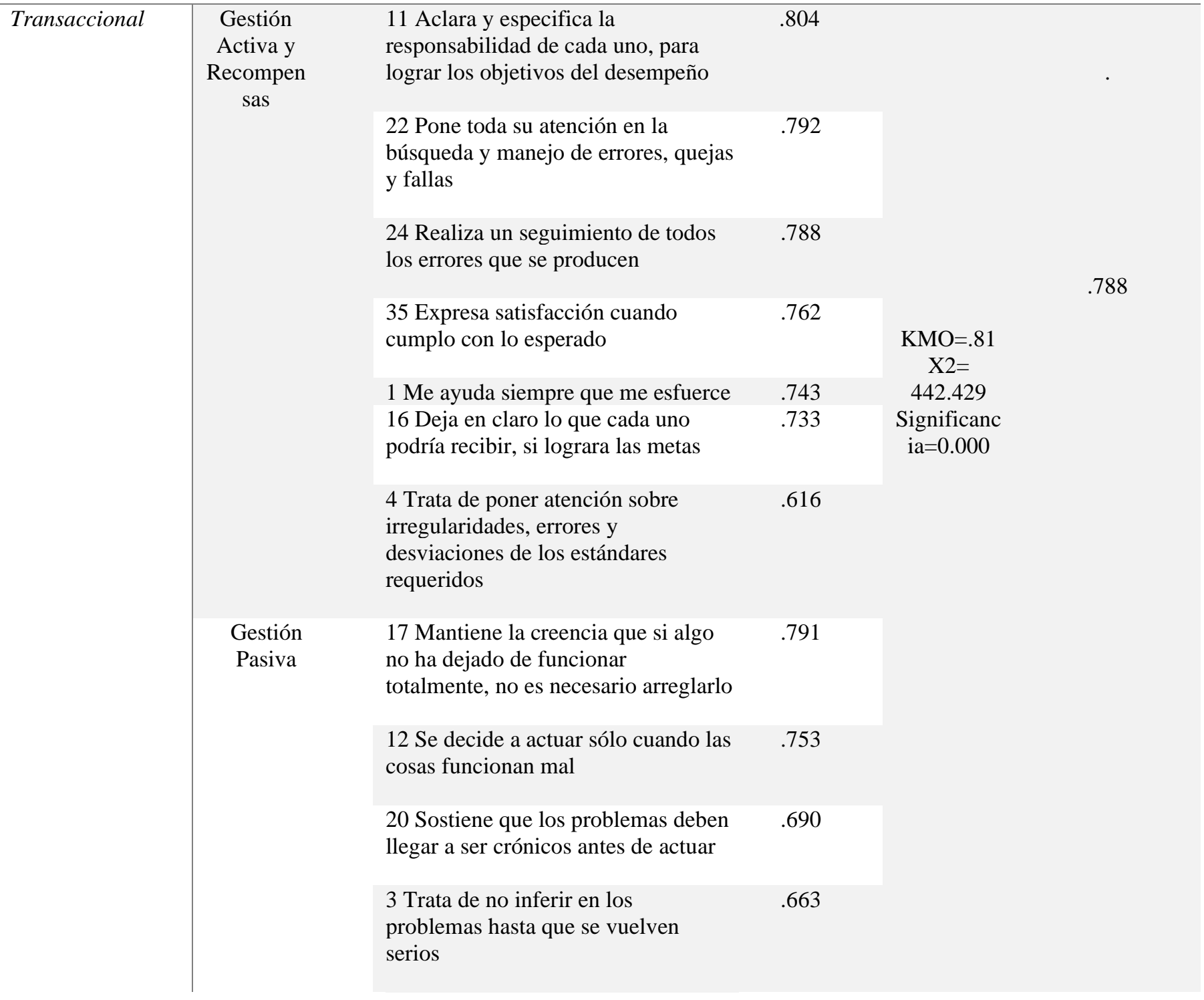

Fuente: Elaboración propia a partir de los datos obtenidos. 


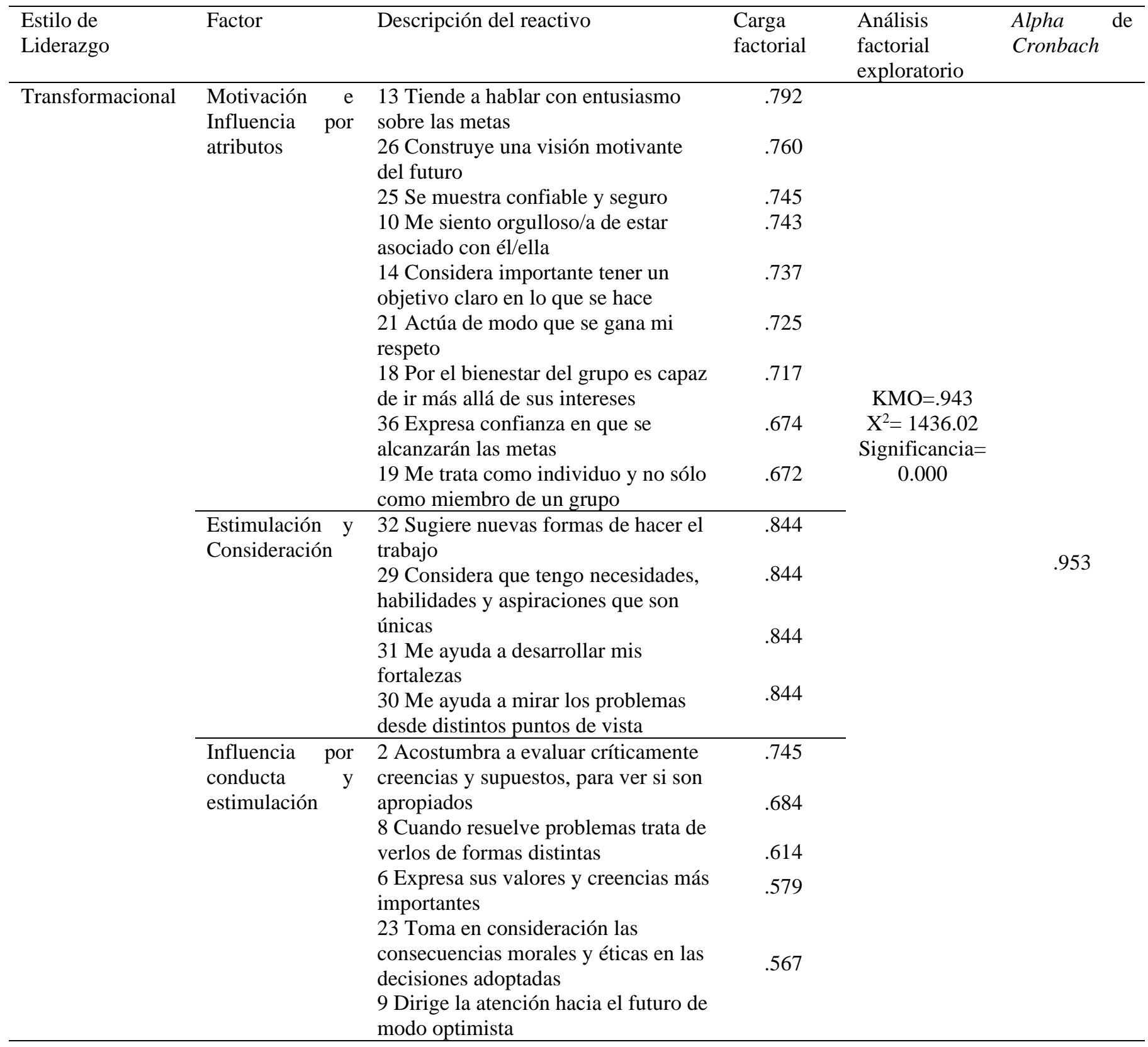

Tabla 3. Estructura del estilo de liderazgo: transformacional.

Fuente: Elaboración propia a partir de los datos obtenidos. 
identificaron solamente dos factores a diferencia de lo planteado en la literatura se

\begin{tabular}{|c|c|c|c|c|c|}
\hline $\begin{array}{r}\text { Estilo de } \\
\text { Liderazgo }\end{array}$ & Factor & Descripción del reactivo & $\begin{array}{l}\text { Carga } \\
\text { factorial }\end{array}$ & $\begin{array}{l}\text { Análisis } \\
\text { factorial } \\
\text { exploratorio }\end{array}$ & $\begin{array}{l}\text { Alpha de } \\
\text { Cronbach }\end{array}$ \\
\hline \multirow[t]{4}{*}{ Laissez-faire } & \multirow[t]{4}{*}{ Laissez-faire } & 28 Le cuesta tomar decisiones & .796 & \multirow{4}{*}{$\begin{array}{c}\mathrm{KMO}=.717 \\
\mathrm{X}^{2}=92.515 \\
\text { Significanci } \\
\mathrm{a}=0.000\end{array}$} & \multirow[t]{4}{*}{.739} \\
\hline & & $\begin{array}{l}33 \text { Tiende a demorar la } \\
\text { respuesta de asuntos urgentes }\end{array}$ & .785 & & \\
\hline & & $\begin{array}{l}7 \text { Suele estar ausente cuando } \\
\text { surgen problemas importantes }\end{array}$ & .708 & & \\
\hline & & $\begin{array}{l}5 \text { Le cuesta involucrarse cuando } \\
\text { surge alguna situación relevante }\end{array}$ & .705 & & \\
\hline
\end{tabular}

Tabla 4. Estructura del estilo de liderazgo: Laissez-faire

Fuente: elaboración propia a partir de los datos obtenidos

\section{CONCLUSIONES}

Del objetivo planteado de la presente investigación de identificar los estilos de liderazgos y sus componentes en organizaciones públicas de Ciudad Victoria, Tamaulipas, cabe mencionar que se pudo cumplir con el mismo al utilizar el instrumento adecuado para la técnica de recolección de datos, así como las herramientas estadísticas pertinente para el tipo de estudio según las validaciones efectuadas.

Los estilos de liderazgo que fueron base para la realización de esta investigación fueron los propuestos por Bernard Bass y Bruce Avolio, en su teoría de liderazgo, propuesta en el año de 1990. Los hallazgos fueron relevantes ya que se observa que la percepción de los encuestados demuestra que en las organizaciones públicas de Ciudad Victoria se identifican los tres estilos de liderazgos los cuales son transformacional, transaccional y laissez faire, Transaccional y Transformacional, así como las estructuras subyacentes de cada uno de ellos.

De los tres estilos de liderazgos el que más predomina es el transaccional el cual se agruparon la gestión activa y la recompensa (especifica la responsabilidad de cada uno, realiza seguimiento de los errores, deja en claro la recompensan que se podría recibir si logra las metas) y el otro en la gestión pasiva (decide actuar solo si no funcionan las cosas, es pasivo ante la necesidad de arreglar algo y solamente actúa si el problema es crónico).

Otro estilo de liderazgo que se identifica es el transformacional, el cual se obtuvo una estructura de tres componentes. El primer componente agrupa aspectos de Motivación e Influencia por atributos, en el que se refleja aspectos de los líderes del sector público de Ciudad Victoria como trabajan sobre las metas a futuro, muestran confianza y seguridad a sus seguidores, los cuales manifiestan el respeto hacia el líder.

Cabe mencionar que el aspecto de estimulación intelectual se distribuyó entre los otros dos factores, en lo referente al de Estimulación y Consideración estimula por medio de nuevas formas para realizar el trabajo considerando las fortalezas de los empleados, como las habilidades y aspiraciones. En la Estimulación que se percibe al realizar la Influencia por conducta muestra aspectos de evaluar con rigidez las creencias y supuestos, toma en consideración 
las consecuencias morales y éticas.

La no existencia de liderazgo o más bien el llamado en la literatura como laissez-faire se agruparon en una sola dimensión por lo que aún existen rasgos como que a algunos líderes les cuesta trabajo tomar decisiones, tienden a demorar las respuestas de asuntos urgente, se ausentan ante asuntos importantes y y les cuesta trabajo involucrarse ante situaciones relevantes. En este aspecto es importante que las organizaciones públicas dadas su razón de ser se analicen estos aspectos porque a pesar de que no se pueden generalizar a la administración pública sin embargo es un acercamiento a considerar dado los resultados obtenidos en cuanto a la confiabilidad de la escala que se utilizó.

\section{REFERENCIAS}

Alonso, F. M., Saboya, P. R., y Guirado, I. C. (2010). Liderazgo transformacional y liderazgo transaccional: un análisis de la estructura factorial del Multifactor Leadership Questionnaire (MLQ) en una muestra española. Psicothema, 22(3), 495-501.

Aguirre, G., Serrano, B., y Sotomayor, G. (2017). El liderazgo de los gerentes de las Pymes de Machala. Universidad y Sociedad, 9(1), 187-195.

Almirón, V., Tikhomirova, A., Trejo, A.C. y García, J.M. (2015). Liderazgo transaccional vs. liderazgo transformacional. ReiDoCrea, 4, 24-27.

Antonakis, J., Avolio, B. J., y Sivasubramaniam, N. (2003). Context and leadership: An examination of the nine-factor full-range leadership theory using the Multifactor Leadership Questionnaire. The leadership quarterly, 14(3), 261-295.

Barahona, H., Cabrera, D. y Torres, U. (2011). Los líderes en el siglo XXI. Entramado, 7(2), 8697.

Bass, B. M., y Avolio, B. (1990). Transformational leadership development: manual for the multifactor leadership questionnaire. Palo Alto, Calif: Consulting Psychologists Press.

Bass, B. M. (1985). Leadership and Performance Beyond Expectations. New York: A Division Of Macmillan.

Bass, B. M. (1991). From transactional to transformational leadership: Learning to share the vision. Organizational dynamics, 18(3), 19-31.

Bass, B. M., y Avolio, B. J. (1997). Full range leadership development: Manual for the Multifactor Leadership Questionnaire (pp. 43-44). Palo Alto, CA: Mind Garden.

Bass, B. M., y Riggio, R. E. (2006). Transformational leadership. Psychology Press.

Bernal, C. (2010). Metodología de la investigación: para administración, economía, humanidades y ciencias sociales. Colombia: Pearson educación.

Blake, R. y Mouton, J. (1964). The Managerial Grid: The key to leadership excellence. Houston: Gulf.

Cruz, V., Salanova, M. y Martínez, I. M. (2013). Liderazgo transformacional: investigación actual y retos futuros. Universidad \& Empresa, 25, 13-32.

Cuadras, C. M. (2007). Nuevos métodos de análisis multivariante. Barcelona: CMC Editions.

Dinwoodie, D., Pasmore, W., Quinn, L. y Rabin, R. (2015). Navigating Change: A Leader's Role. Center for Creative Leadership.

Eagly, A. H., Johannesen-Schmidt, M. C., y Van Engen, M. L. (2003). Transformational, transactional, and laissez-faire leadership styles: a meta-analysis comparing women and men. Psychological bulletin, 129(4), 569. 
Enderica, O., D’Armas, M., Bermeo, R. J., López, M., Tinoco, E. y Carrión, G. (2018). Buen liderazgo, una experiencia en el crecimiento empresarial de las PYMES. Espacios, 39(42), 4-14.

Escandón, D. M., y Hurtado, A. (2016). Influencia de los estilos de liderazgo en el desempeño de las empresas exportadoras colombianas. Estudios Gerenciales, 32(139), 137-145.

Fernández, S. (2014). Un análisis del fenómeno del liderazgo en la empresa. (Tesis de pregrado). Universidad de León: España.

García, M. (2011). Liderazgo transformacional y la facilitación de la aceptación al cambio organizacional. Pensamiento Psicológico, 9(16), 41-54.

García, M. (2015). Formulación de un modelo de liderazgo desde las teorías organizacionales. Entramado, 11(1), 60-79.

George, D., \& Mallery, P. (2003). SPSS for Windows step by step: A simple guide and reference. 11.0 update (4thed.). Boston: Allyn \& Bacon

González, E. L., Carrión, A. y Palacios, D. (2015). El liderazgo por competencias y el EFQM. Investigación administrativa, 44(116), 1-25.

Gómez, R. A. (2006). El liderazgo empresarial para la innovación tecnológica en las micro, pequeñas y medianas empresas. Universidad y Empresa, 5(11), 62-91.

Gómez, J.G.I. (2016). El liderazgo de aprendizaje y su incidencia en la innovación y competitividad de las PyMEs de la Ciudad de Durango, México. Investigación Administrativa, 45(117), 52-67.

Hair, J. F., Anderson, R. E., Tatham, R. L., \& Black, W. C. (1999). Análisis multivariante. Madrid: Prentice Hall.

Hernández, Y., Hernández, G. y Mendieta, A. (2013) Modelo de rotación de personal y prácticas organizacionales. Historia y Comunicación Social. 18, 837-863. https://doi.org/10.5209/rev_HICS.2013.v18.44369

Hinkin, T. R., y Schriesheim, C. A. (2008). A theoretical and empirical examination of the transactional and non-leadership dimensions of the Multifactor Leadership Questionnaire (MLQ). The Leadership Quarterly, 19(5), 501-513.

Horwitz, I. B., Horwitz, S. K., Daram, P., Brandt, M. L., Brunicardi, F. C., y Awad, S. S. (2008). Transformational, transactional, and passive-avoidant leadership characteristics of a surgical resident cohort: analysis using the multifactor leadership questionnaire and implications for improving surgical education curriculums. Journal of surgical Research, 148(1), 49-59.

Koontz, H., y Weihrich, H. (1998). Administración: una perspectiva global (Vol. 11). McGraw-Hill. Liu, J., Siu, O. L., y Shi, K. (2010). Transformational Leadership and Employee Well-Being: The Mediating Role of Trust in the Leader and Self-Efficacy. Applied Psychology, 59(3), 454479.

Ma, L., y Tsui, A. S. (2015). Traditional Chinese philosophies and contemporary leadership. The Leadership Quarterly, 26(1), 13-24.

Lupano, M., y Castro, A. (2006). Estudios sobre el liderazgo - Teorías y evaluación. Psicodebate. Psicología, Cultura y Sociedad, 6, 107-122.

Noriega, M. G. (2008). La importancia del liderazgo en las organizaciones. Temas de Ciencia y Tecnología, 12(36), 25-29.

Perdomo, Y. y Prieto, R. (2009). El liderazgo como herramienta de competitividad para la gerencia de servicio. Centro de Investigación de Ciencias Administrativas y Gerenciales, 6(2), 1931.

Rivera, Y. M. y Conrado, J. (2016). Impacto de los líderes en la productividad de las empresas de servicio de aseo en la ciudad de Barranquilla. Dictamen Libre, 19, 57-68.

Robbins, S. Coulter, M. (2010). Administración (10ª ed.). (L. Fernández \& V. Alba, Trads). México: Pearson. (Trabajo original publicado en 2009).

Sosik, J. J.y Dione, S. D. (1997). Leadership Styles and Deming's Behavior Factors. Journal of 
Business and Psychology, 11 (4): 447-462

Stogdill, R. M. (1948). Personal factors associated with leadership: A survey of the literature. Journal of Psychology, 25, 35-71.

Tracy, B. (2014) Liderazgo. México: Grupo Nelson.

Vos, J. y Rupert, J. (2018). Change agent's contribution to recipient's resistance to change: A two sided story. European Management Journal, 36(4), 453-462.

Yukl, G. (1999) An Evaluation of the Conceptual Weaknesses in Transformational and Charismatic Leadership Theories. Leadership Quarterly. 10(2), 285-305. 
